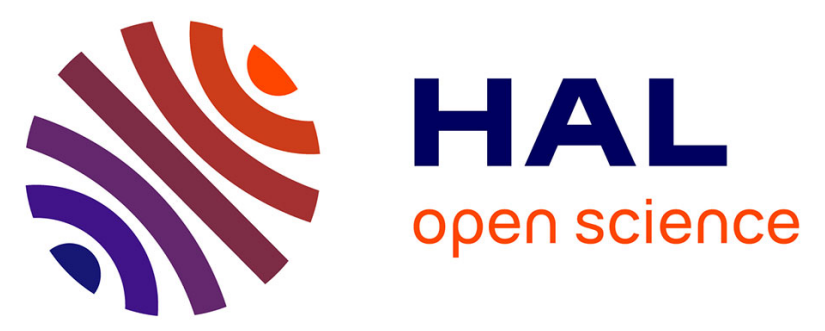

\title{
Méthode de mesure directe de coefficients dynamiques d'induction magnétique. Application dans le cas de bobinages supraconducteurs avec carcasse ferromagnétique \\ D. Genotel, M. Girard, G. Balossier
}

\section{To cite this version:}

D. Genotel, M. Girard, G. Balossier. Méthode de mesure directe de coefficients dynamiques d'induction magnétique. Application dans le cas de bobinages supraconducteurs avec carcasse ferromagnétique. Revue de Physique Appliquée, 1977, 12 (6), pp.961-968. 10.1051/rphysap:01977001206096100 . jpa00244267

\section{HAL Id: jpa-00244267 https://hal.science/jpa-00244267}

Submitted on 1 Jan 1977

HAL is a multi-disciplinary open access archive for the deposit and dissemination of scientific research documents, whether they are published or not. The documents may come from teaching and research institutions in France or abroad, or from public or private research centers.
L'archive ouverte pluridisciplinaire HAL, est destinée au dépôt et à la diffusion de documents scientifiques de niveau recherche, publiés ou non, émanant des établissements d'enseignement et de recherche français ou étrangers, des laboratoires publics ou privés. 


\title{
MÉTHODE DE MESURE DIRECTE DE COEFFICIENTS DYNAMIQUES D'INDUCTION MAGNÉTIQUE APPLICATION DANS LE CAS DE BOBINAGES SUPRACONDUCTEURS AVEC CARCASSE FERROMAGNÉTIQUE
}

\author{
D. GENOTEL, M. GIRARD et G. BALOSSIER \\ Laboratoire de Microscopie Electronique (*), Université de Reims, U. E. R. Sciences \\ B. P. no 347, 51062 Reims Cedex, France
}

(Reçu le 25 novembre 1976, révisé le 15 février 1977, accepté le 2 mars 1977)

\begin{abstract}
Résumé. - Nous rappelons tout d'abord la distinction entre les coefficients statiques et les coefficients dynamiques d'induction pour des circuits qui en présence de carcasses ferromagnétiques ont une réponse en flux non linéaire. Nous proposons alors une méthode de mesure qui permet de déterminer les coefficients dynamiques d'induction plus directement que les méthodes balistiques habituelles. Nous l'appliquons à la mesure des coefficients dynamiques d'auto-induction de lentilles électroniques magnétiques à enroulements excitateurs supraconducteurs : nous étudions ces coefficients en fonction de l'excitation des lentilles et pour diverses lentilles semblables. La méthode est cependant plus générale et peut être appliquée à la mesure d'inductances quelconques en particulier dans le domaine industriel, pour des machines électriques ou des transformateurs.
\end{abstract}

\begin{abstract}
We first recall the distinction between secant and incremental inductances of windings with a ferromagnetic core. A method of measurement which allows direct determination of incremental inductances is then discribed and compared with conventional techniques. Its application is illustrated by measurements made on superconducting magnetic electron-lenses : their incremental inductances are measured as functions of the excitation for some geometrically similar lenses. The method is moreover applicable to the measurement of comparatively larger inductances and to industrial circuits which carry heavy currents such as large electrical machines or transformers.
\end{abstract}

1. Introduction. - Dans le microscope électronique supraconducteur $400 \mathrm{kV}$ que nous avons construit [1] les enroulements excitateurs des lentilles magnétiques sont réalisés en fil supraconducteur. Ces enroulements excitateurs refermés sur eux-mêmes fonctionnent en boucle fermée (Fig. 1) et leur stabilité en courant est

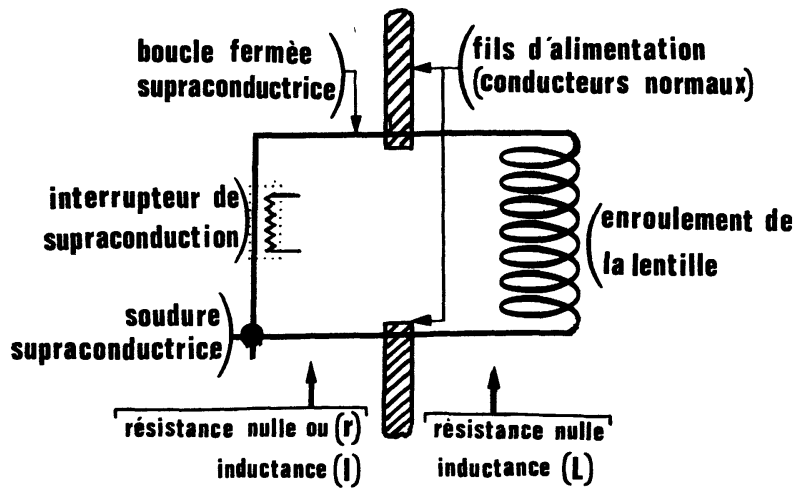

FIG. 1. - Schéma d'un circuit supraconducteur en boucle fermée. [Superconducting circuit in the persistent mode.]

(*) Equipe de Recherche associée au C. N. R. S. n 376. parfaite : des interrupteurs de supraconduction permettent l'établissement et le piégeage de courants électriques dans ces enroulements [2]. Dans ces conditions l'établissement d'un courant se fait avec une constante de temps $\tau=L^{\prime} / r$ ou $L^{\prime}$ est le coefficient d'autoinduction de la lentille et $r$ la résistance introduite par son interrupteur. Pour évaluer le temps d'établissement d'un courant il est donc nécessaire de connaître la valeur de ce coefficient d'auto-induction $L^{\prime}$ de la lentille.

D'autre part pour effectuer la mise au point ou pour réaliser une série focale on doit pouvoir commander avec précision des petites variations de courant $\Delta I$ dans la lentille objectif. Nous avons montré [2] qu'il était préférable d'induire ces petites variations avec un transformateur à courant continu. La variation de flux $\Delta \Phi$ que doit créer le transformateur est donnée en fonction de la variation de courant $\Delta I$ par la formule $\Delta \Phi=L^{\prime} . \Delta I$. Il est donc également nécessaire de connaître $L^{\prime}$ pour prévoir l'action du transformateur et pour le calculer en conséquence.

Nous sommes donc amenés à envisager la mesure du coefficient dynamique d'auto-induction d'une lentille magnétique à enroulement supraconducteur. Dans 
différents articles [3], [4], [5] traitant de la mesure de coefficient d'auto-induction ou d'induction mutuelle de circuits électriques les auteurs ne font pas toujours de distinction nette entre coefficients statiques et coefficients dynamiques pas plus d'ailleurs qu'entre les mesures expérimentales qui s'y rapportent. Cette distinction étant fondamentale pour les circuits qui ont, comme nos lentilles, une réponse en flux non linéaire, nous rappellerons ici les définitions de ces coefficients statiques et dynamiques que l'on notera respectivement $L$ et $M$ d'une part, $L^{\prime}$ et $M^{\prime}$ d'autre part.

Nous examinerons alors les diverses techniques de mesure de coefficients d'induction en précisant les cas où elles s'appliquent et la nature des coefficients qu'elles permettent de mesurer. Aucune des méthodes classiques n'étant applicables sans inconvénient au cas d'un enroulement supraconducteur nous présentons finalement une technique de mesure originale particulièrement adaptée à ce cas et permettant de plus une mesure directe des coefficients dynamiques.

2. Définitions des coefficients statiques et dynamiques d'induction. - 2.1 COEFFICIENTS D'AUTOINDUCTION D'UN CIRCUIT. - Soit $I$ l'intensité du courant électrique qui parcourt un circuit et $\Phi$ le flux magnétique qui le traverse : le coefficient statique d'auto-induction de ce circuit est le rapport entre le flux et l'intensité du courant soit $L=\Phi / I$ cependant que le coefficient dynamique représente le taux de variation de ce flux avec l'intensité soit $L^{\prime}=\mathrm{d} \Phi / \mathrm{d} I$.

Avec un seul circuit on a une seule variable intensité $I$ et les coefficients $L$ et $L^{\prime}$ ci-dessus peuvent toujours être définis quelles que soient les propriétés magnétiques du milieu environnant. Quand le circuit est dans un milieu de perméabilité magnétique constante sa caractéristique $\Phi(I)$ est linéaire (Fig. $2 a$ ) et les deux coefficients ont une même valeur constante $L=L^{\prime}=$ cte. Par contre quand le milieu comporte des éléments ferromagnétiques de perméabilité variable sa réponse en flux n'est plus linêaire (Fig. $2 b$ ) et les

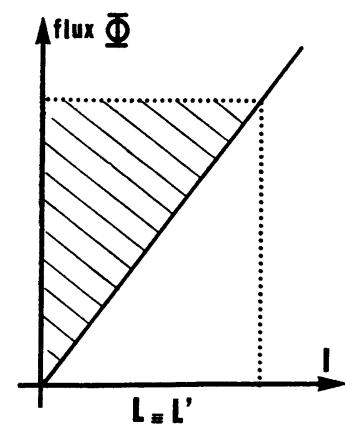

[a]

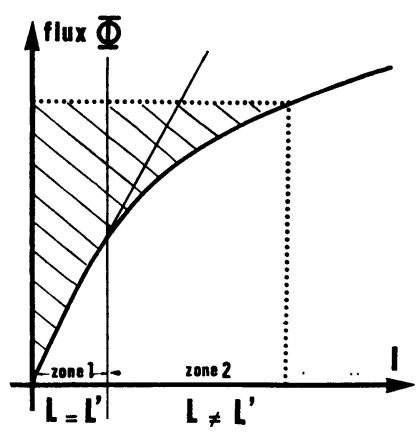

$[$ b]
FIG. 2. - Courbe de flux magnétique propre d'un circuit électrique en fonction de son courant d'excitation. $(a)$ : réponse linéaire; $(b)$ : réponse non linéaire.

[Magnetic flux characteristic of an electrical circuit. (a) : linear response ; (b) : non-linear response.] coefficients statique et dynamique sont alors différents et tous deux fonctions de l'intensité du courant :

$$
L(I) \neq L^{\prime}(I)
$$

L'énergie magnétique stockée dans un tel circuit a pour expression

$$
W=\int_{0}^{\Phi} I \cdot \mathrm{d} \Phi
$$

et correspond à la surface hachurée sur la caractéristique $\Phi(I)$ (Fig. 2). Quand cette caractéristique est linéaire l'énergie a la forme bien connue

$$
W=\Phi \cdot I / 2=L . I^{2} / 2 .
$$

Par contre quand le circuit ne répond pas linéairement l'énergie stockée est inférieure à $\Phi . I / 2$ et on ne peut l'évaluer qu'en déterminant exactement la caractéristique $\Phi(I)$ ce qui conduit aux mêmes techniques de mesure que pour la détermination de $L(I)$ ou $L^{\prime}(I)$.

2.2 COEFFICIENTS D'INDUCTION DE PLUSIEURS CIRCUITS. - Nous raisonnerons sur le cas simple de deux circuits $\left(\mathrm{C}_{1}\right)$ et $\left(\mathrm{C}_{2}\right)$ : on a alors un problème à deux variables et les flux magnétiques $\Phi_{1}$ et $\Phi_{2}$ des circuits dépendent tous deux des deux intensités $I_{1}$ et $I_{2}$.

Quand la perméabilité du milieu environnant est constante, les flux sont des combinaisons linéaires de $\boldsymbol{I}_{\mathbf{1}}$ et $I_{2}$ soit par exemple pour le circuit $\left(\mathrm{C}_{1}\right)$

$$
\Phi_{1}\left(I_{1}, I_{2}\right)=\alpha . I_{1}+\beta I_{2} .
$$

On peut donc distinguer le flux partiel d'auto-induction $\Phi_{11}=\alpha . I_{1}$ et le flux partiel d'induction mutuelle $\Phi_{12}=\beta . I_{2}$ et définir avec eux des coefficients statiques d'induction en formant les rapports de ces flux partiels et des intensités correspondantes soit :

$$
L_{1}=\Phi_{11} / I_{1}=\alpha \quad \text { et } \quad M_{12}=\Phi_{12} / I_{2}=\beta .
$$

Les coefficients dynamiques d'induction sont les dérivées partielles des flux des circuits ; dans le cas présent ils ont les mêmes valeurs que les coefficients statiques soit pour le circuit $\left(\mathrm{C}_{1}\right)$ :

$$
L_{1}^{\prime}=\partial \Phi_{1} / \partial I_{1}=\alpha \quad \text { et } \quad M_{12}^{\prime}=\partial \Phi_{1} / \partial I_{2}=\beta .
$$

Par contre quand le milieu a une perméabilité variable les flux ne sont plus linéaires en $I_{1}$ et $I_{2}$. On ne peut plus distinguer de flux partiels et on ne peut donc plus définir de coefficients statiques d'induction : seuls les coefficients dynamiques ont encore une signification.

3. Coefficients d'auto-induction d'une lentille électronique magnétique avec fer. - Une lentille magnétique avec fer est constituée d'un enroulement excitateur et d'un circuit ferromagnétique avec entrefer (Fig. 3). La caractéristique $\Phi(I)$ d'une telle lentille a une allure analogue à celle de la figure $2 b$ et on peut y distinguer deux zones.

Dans la zone 1 aux faibles excitations la réluctance constante de l'entrefer est prépondérante et on y 


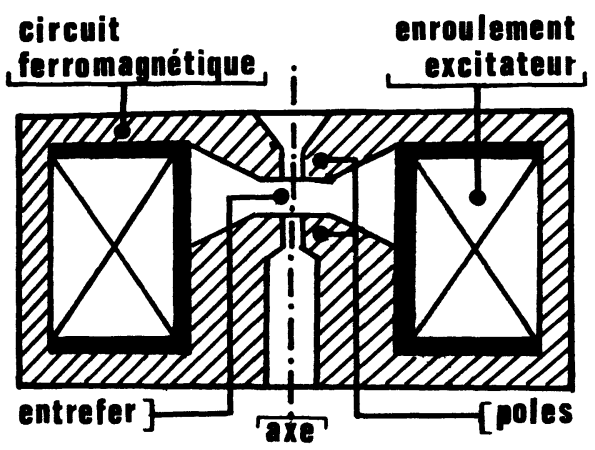

Fig. 3. - Coupe méridienne schématique d'une lentille électronique magnétique avec carcasse ferromagnétique.

[Cross-section of a typical magnetic electron-lens with an iron core.]

retrouve toute la force magnétomotrice de l'enroulement excitateur ce qui linéarise la réponse en flux de la lentille. Dans cette zone les coefficients statique et dynamique d'auto-induction sont donc égaux et constants malgré la présence de la carcasse en fer : $L \equiv L^{\prime}=L_{1}$. Les lentilles magnétiques traditionnelles dont les enroulements excitateurs sont en cuivre, fonctionnent souvent dans cette première zone.

Dans la zone 2 apparaît le phénomène de saturation du circuit magnétique dont la réluctance n'est plus négligeable et s'ajoute à celle de l'entrefer pour infléchir la caractéristique $\Phi(I)$ : à la limite la réponse en flux de la lentille tend vers celle de l'enroulement excitateur seul. Le coefficient statique $L$ diminue lentement cependant que le coefficient dynamique $L^{\prime}$ décroît très vite pour approcher rapidement sa limite $L_{\mathrm{p}}$ auto-inductance propre de la bobine excitatrice. Avec les lentilles à enroulements supraconducteurs on travaille souvent en régime saturé dans cette seconde zone.

$\mathrm{Si}$ on utilise des lentilles supraconductrices fonctionnant en boucle fermée il est nécessaire, comme nous l'avons dit dans l'introduction, de connaître la valeur de leurs coefficients d'induction qui interviennent dans le temps de charge en courant et dans l'induction de petites variations permanentes de courant au voisinage de l'intensité qui est piégée.

4. Méthodes de mesure des coefficients d'induction. Il existe différentes méthodes de mesure [6] qui ne sont pas toujours applicables dans tous les cas selon par exemple que le circuit considéré est ou n'est pas supraconducteur ou que sa réponse en flux est ou n'est pas linéaire : on peut les classer en deux groupes, celui des mesures en alternatif et celui des mesures balistiques.

4.1 Méthodes De Mesure en Alternatif. - Dans ce premier cas on travaille en régime permanent sinusoïdal et on mesure l'impédance des inductances soit par une mesure courant-tension soit surtout à l'aide de ponts de mesure. Ces mesures au pont sont applicables dans le cas d'inductances assez faibles utilisées dans les circuits radioélectriques. Elles s'appli- quent quand les circuits ont une réponse linéaire en flux, les inductances statique et dynamique ayant alors la même valeur constante : c'est le cas principalement pour des bobines sans noyaux ferromagnétiques. Pour des bobines avec noyaux non saturés la réponse en flux est également linéaire mais il y a lieu de faire attention car l'inductance apparente mesurée en alternatif est fonction de la fréquence à cause des courants de Foucault.

Ces mesures en alternatif ne sont par contre pas applicables dans le cas de circuits comportant des éléments ferromagnétiques qui se saturent, ce qui est le cas de nos lentilles : la réponse en flux n'est en effet plus linéaire et l'interprétation de la mesure effectuée est alors délicate. Ces mesures ne s'appliquent pas non plus à des circuits supraconducteurs dont les caractéristiques doivent être étudiées quand ils sont parcourus par des courants de forte intensité, ce qui est encore le cas de nos lentilles. On doit en effet éviter les variations trop rapides de courants intenses dans les fils supraconducteurs surtout dans les fils monobrin car dans ces conditions les pertes qui apparaissent dans les fils risquent de les faire transiter localement à l'état normal entraînant alors leur détérioration.

4.2 MÉThOdes DE MESURE BALISTIQUes. - Les méthodes balistiques utilisent des courants continus et font intervenir les régimes transitoires d'établissement ou de variation de ces courants. Elles consistent à mesurer des flux ou des variations de flux à l'aide d'un intégrateur galvanométrique ou électronique qui intègre dans le temps $t$ une tension instantanée $e=\mathrm{d} \Phi / \mathrm{d} t$ induite dans un circuit.

Dans une première méthode on peut utiliser une bobine auxiliaire pour détecter les flux à mesurer mais cela présente plusieurs inconvénients :

- d'une part, il n'est pas toujours possible de loger convenablement la bobine de mesure dans le circuit étudié,

- d'autre part, on ne connaît pas toujours le nombre de tours du circuit étudié,

- enfin, les mesures qu'on peut effectuer sont approchées car le flux traversant la bobine de mesure ne concerne pas nécessairement toutes les spires du circuit considéré.

La seconde méthode consiste à disposer l'intégrateur aux bornes du circuit étudié qui sert alors lui-même de bobine de mesure. Si on mesure un flux d'induction extérieur dans un circuit $\left(C_{1}\right)$ qui n'est pas lui-même alimenté il n'y a pas de tension continue à ses bornes et on peut y placer directement l'intégrateur (Fig. 4). Par contre si on mesure le flux propre de ce circuit ou si on mesure un flux d'induction extérieur, ce circuit étant lui-même alimenté on doit alors utiliser un montage en pont de Maxwell-Rayleigh pour éliminer la composante de tension continue due à la résistance $R_{1}$ du circuit considéré (Fig. 5). Si on note $e_{1}$ la tension induite dans la bobine étudiée parcourue par un cou- 


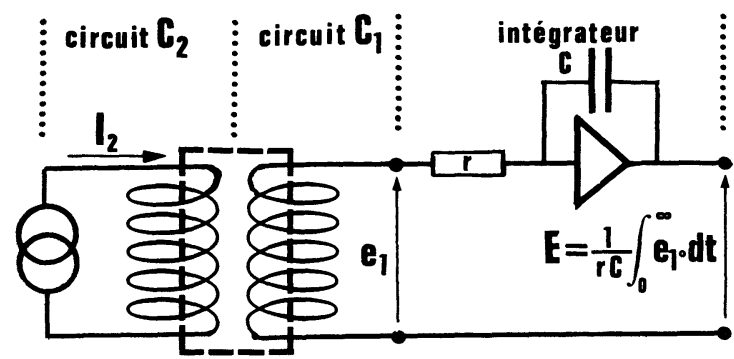

Fig. 4. - Montage pour la mesure du flux $\Phi_{21}$ envoyé par le circuit $C_{2}$ dans le circuit $C_{1}$ non alimenté.

[Circuit for the measurement of the mutual induction magnetic flux with $\left(\mathrm{C}_{1}\right)$ and $\left(\mathrm{C}_{2}\right)$ windings, $\left(\mathrm{C}_{1}\right)$ not supplied.]

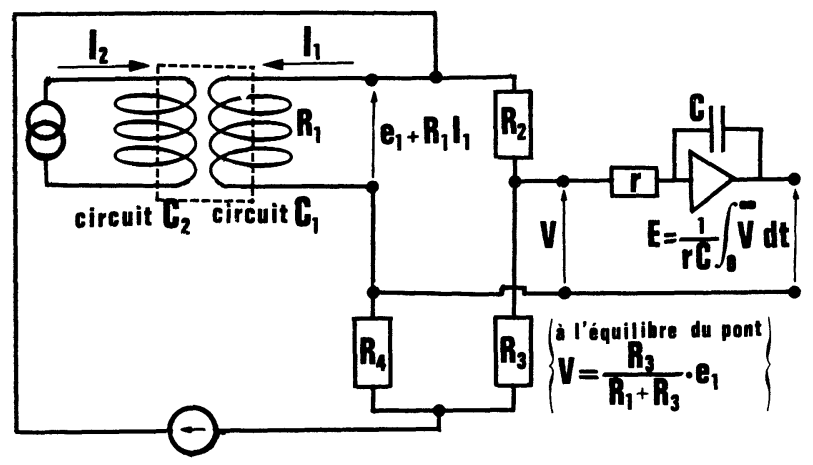

Fig. 5. - Montage en pont de Maxwell-Rayleigh pour la mesure du flux propre $\Phi_{11}$ du circuit $C_{1}$ ou de flux $\Phi_{21}$ envoyé par le circuit $C_{2}$ dans le circuit $C_{1}$ lui même alimentée.

[Maxwell-Rayleigh bridge circuit for the measurement of the self and mutual induction magnetic fluxes, with $\left(\mathrm{C}_{1}\right)$ and $\left(\mathrm{C}_{2}\right)$ windings both being supplied.]

rant $I_{1}$ on retrouve sur la diagonale du pont de tension :

$$
V_{1}=e_{1} \cdot \frac{R_{3}}{R_{2}+R_{3}}-I_{1} \cdot \frac{R_{2} R_{4}-R_{1} R_{3}}{R_{2}+R_{3}} .
$$

Si on équilibre le pont pour avoir $R_{1} R_{3}=R_{2} R_{4}$ on a alors sur sa diagonale une tension proportionnelle à $e_{1}$ et on peut y placer l'intégrateur.

Dans ces méthodes balistiques on doit donc effectuer des variations de courant dans les circuits et mesurer les variations de flux correspondantes. Habituellement avec des circuits normaux on opère de façon simple en ouvrant par exemple le circuit d'alimentation de l'enroulement considéré ce qui revient à décharger très rapidement l'énergie de l'enroulement dans l'étincelle de rupture. Avec des circuits supraconducteurs on ne doit pas effectuer de variations de courant aussi rapides. Dans une décroissance exponentielle avec une constante de temps $\tau$ la vitesse de variation du courant $d I / \mathrm{d} t$ est égale à $I / \Phi$. Pour réduire cette vitesse sans modifier l'excitation du circuit on doit donc augmenter la constante de temps en déchargeant les bobines supraconductrices dans une résistance très faible. On peut alors opérer en utilisant le montage simple présenté sur la figure 6 .

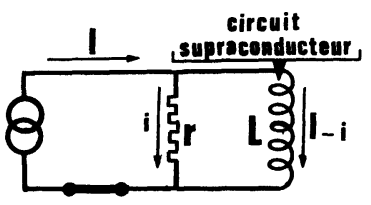

(a)

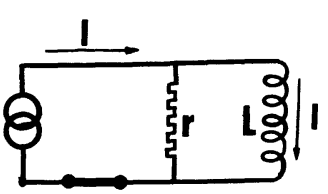

(b) (c)

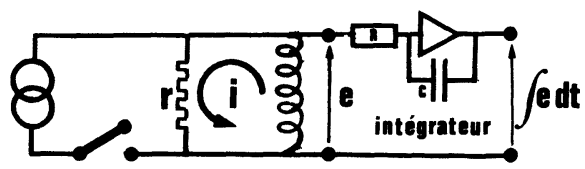

FIG. 6. - Montage pour la mesure du flux propre d'un circuit supraconducteur déchargé dans une faible résistance. $(a)$ et $(b)$ : charge du circuit; (c) : décharge du circuit.

[Circuit to measure the magnetic flux of a superconducting winding discharged in a weak resistance. $(a)$ and $(b)$ : loading of the winding ; $(c)$ : discharge in the resistance.]

Ces mesures balistiques qui procèdent par intégration donnent accès à des flux et permettent de déterminer des caractéristiques $\Phi(I)$ et donc des coefficients statiques d'induction. Quand la caractéristique $\Phi(I)$ n'est pas linéaire, l'accès aux coefficients dynamiques d'induction nécessite une évaluation de la pente de cette courbe. Pour nos lentilles supraconductrices qui sont dans ce cas, nous avons donc défini et utilisé une méthode de mesure originale simple dans laquelle on contrôle également la vitesse de variation du courant et qui de plus, permet la détermination directe des coefficients dynamiques d'induction.

5. Méthode de mesure directe des coefficients dynamiques d'induction. - Cette méthode spécialement introduite pour des circuits supraconducteurs ayant une réponse en flux non linéaire est cependant applicable dans tous les cas. Nous en présentons ici le principe et l'application dans le cas simple de la mesure du coefficient dynamique d'autoinduction d'un circuit.

5.1 Principe de mesure. - A une variation de courant $\mathrm{d} I$ dans un circuit isolé correspond une variation de flux $\mathrm{d} \Phi$ et une tension induite $e=\mathrm{d} \Phi / \mathrm{d} t$ qu'on peut mettre sous la forme $e=L^{\prime} . \mathrm{d} I / \mathrm{d} t$, le coefficient $L^{\prime}=\mathrm{d} \Phi / \mathrm{d} I$ étant le coefficient dynamique d'autoinduction du circuit. La méthode consiste à alimenter le circuit avec un générateur de courant en forme de rampe qui impose une valeur $\mathrm{d} I / \mathrm{d} t=a$ constante (Fig. 7) et à déterminer expérimentalement

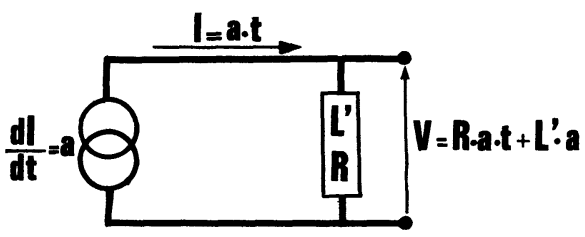

FIG. 7. - Montage pour la mesure du coefficient dynamique d'autoinduction d'un circuit en utilisant une rampe de courant.

[Circuit for the measurement of the incremental self-induction coefficient of a winding supplied by a linear slope current.] 
la force électromotrice induite $e(t)$ : on en déduit alors directement le coefficient dynamique d'induction $L^{\prime}=e / a$. Pour la détermination de $e$ on distinguera le cas simple des circuits supraconducteurs et le cas des circuits résistants.

5.2 CAS D'UN CIRCUIT SUPRACONDUCTEUR. - Quand le circuit est supraconducteur on a directement la tension $e(t)$ aux bornes du circuit : si on enregistre cette tension on obtient une courbe dont l'allure est présentée sur la figure 8 . Au voisinage de l'origine

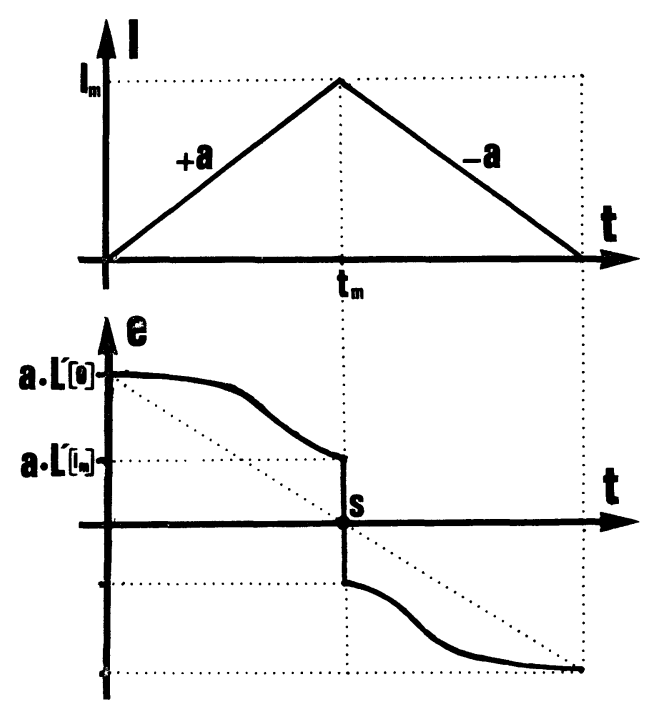

Fig. 8. - Tension $e(t)$ enregistrée aux bornes d'un circuit supraconducteur traversé par la rampe de courant $I= \pm$ a.t.

[Curves giving the current $I(t)$ flowing in a superconducting winding and the voltage $e(t)$ induced in the circuit. ]

la tension $e(t)$ prend presque instantanément la valeur $L^{\prime}(o) . a$. Elle diminue ensuite quand le circuit magnétique se sature ce qui traduit la décroissance de $L^{\prime}$ qui tend vers l'inductance propre $L_{\mathrm{p}}$ de la bobine excitatrice. Au point de basculement de la rampe elle passe instantanément de la valeur $+L^{\prime}\left(I_{\mathrm{m}}\right) \cdot a$ à la valeur $-L^{\prime}\left(I_{\mathrm{m}}\right)$.a. La seconde branche de la courbe qui correspond à la rampe de courant décroissante est symétrique de la première branche par rapport au point $S$ de l'axe des temps et une seule d'entre elles suffit donc pour déterminer $L^{\prime}=e / a$ sur l'intervalle d'intensité de $O$ à $I_{\mathrm{m}}$, Nous présentons dans le paragraphe 6 quelques résultats expérimentaux obtenus dans ces conditions pour nos lentilles supraconductrices.

5. 3 CAS D'UN CIRCUIT RÉSISTANT. - Dans le cas général d'un circuit résistant la tension enregistrée a pour expression $V(t)=e(t)+R \cdot I(t)$. Sur la figure 9 on a représenté en trait pointillé l'allure des termes $e(t)$ et $R . I(t)$ qui dans la pratique ne sont pas connus; en trait plein on donne leur somme qui correspond à la courbe effectivement enregistrée : cette courbe présente toujours deux branches $(\mathrm{AB})$ et $(\mathrm{CD})$ qui

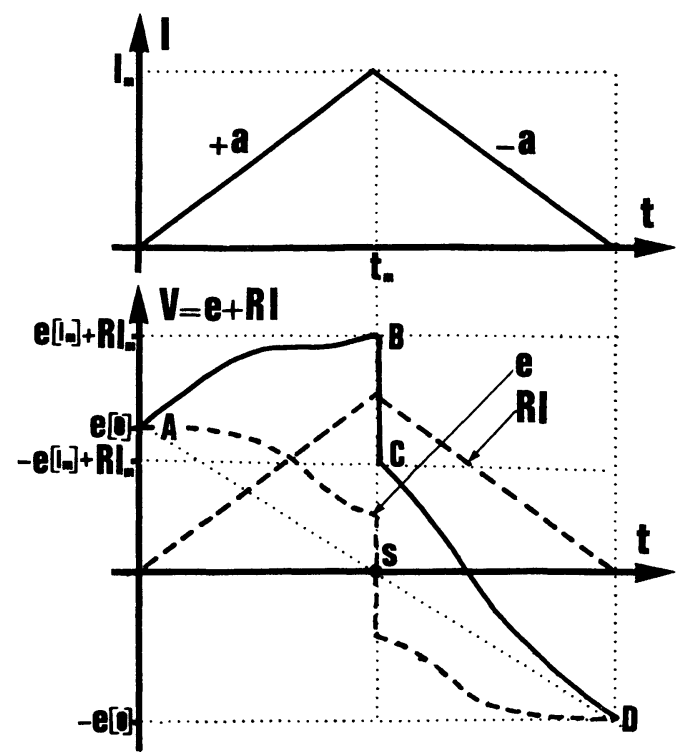

Fig. 9. - Tension $V(t)$ enregistrée aux bornes d'un circuit résistant traversé par la rampe de courant $I= \pm a . t$.

[Curves giving the current $I(t)$ flowing in a resistant winding and the total voltage $V(t)$ measured at the circuit terminals. ]

cette fois ne sont plus symétriques par rapport au point S. Sur la figure 10 on a tracé la courbe (ABCD) et la courbe $\left(D^{\prime} C^{\prime} A\right)$ déduite de la première par symétrie par rapport au point $\mathrm{S}$. Ces deux courbes donnent respectivement $(e+R . I)$ et $(e-R . I)$ : la courbe moyenne qu'on peut déterminer nous donne

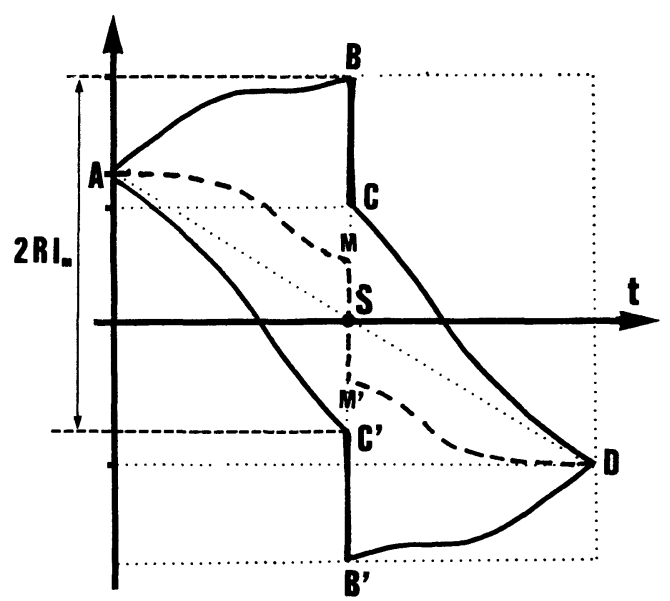

FIG. 10. - Détermination graphique de la tension $e(t)$ (courbe $\left.\mathrm{AMM}^{\prime} \mathrm{D}\right)$ et de la résistance $R$ du circuit $\left(\mathrm{BC}^{\prime}=2 . \mathrm{R} . I_{\mathrm{m}}\right)$ à partir de la tension enregistrée $V(t)$ (courbe $\mathrm{ABCD})$.

[Graphical determination of the induced voltage $e(t)$ (curve $\left.\mathrm{AMM}^{\prime} \mathrm{D}\right)$ and the winding resistance $R\left(\mathrm{BC}^{\prime}=2 . R . I_{\mathrm{m}}\right)$ from the measured total voltage $V(t)$ (curve ABCD).]

donc la tension $e$ que l'on recherche pour calculer le coefficient d'autoinduction du circuit. D'autre part la longueur du segment $\mathrm{BC}^{\prime}$ égale à $2 . R . I_{\mathrm{m}}$ permet la détermination de la résistance $R$ du circuit. 
Il est dans ce cas important de remarquer que si la tension résistive $R I$ est trop importante devant la tension induite $e$, l'enregistrement de la tension totale ne permet plus une détermination précise de cette tension induite. Pour remédier à cela nous pouvons procéder de deux façons différentes :

- La première consiste à augmenter la valeur de $a$ en changeant les caractéristiques de l'intégrateur pour avoir des rampes de courant plus rapides, la commutation pour le basculement de la rampe étant alors électronique et les enregistrements de tension étant faits à l'oscilloscope. On choisit alors un temps de montée $t_{\mathrm{m}}=I_{\mathrm{m}} / a$ petit devant la constante de temps $\tau=L^{\prime} / R$ du circuit.

- La seconde solution consiste à contrebalancer le terme $R I$ à l'aide d'une rampe de tension négative - $b . t$ qu'on ajoute à travers un montage additionneur pour avoir une tension totale voisine de $e$ soit : $V=e+R I-b t \simeq e$. La rampe de tension négative n'a d'ailleurs pas besoin de compenser rigoureusement le terme $R I$ et il suffit que la tension résiduelle $\varepsilon=R I-b t$ soit petite devant $e$, la technique décrite ci-dessus permettant alors de déterminer $e$ quelle que soit la forme de cette tension résiduelle $\varepsilon$.

5.4 UTILISATION DE LA MÉTHODE DANS LE CAS DE PLUSIEURS CIRCUITS. - Considérons par exemple le cas simple où on a deux circuits en présence dont les flux sont $\Phi_{1}\left(I_{1}, I_{2}\right)$ et $\Phi_{2}\left(I_{1}, I_{2}\right)$. Les tensions induites dans ces circuits ont pour expressions :

$e_{1}=\frac{\mathrm{d} \Phi_{1}}{\mathrm{~d} t}=\frac{\partial \Phi_{1}}{\partial I_{1}} \frac{\mathrm{d} I_{1}}{\mathrm{~d} t}+\frac{\partial \Phi_{1}}{\partial I_{2}} \frac{\mathrm{d} I_{2}}{\mathrm{~d} t}=L_{1}^{\prime} \frac{\mathrm{d} I_{1}}{\mathrm{~d} t}+M^{\prime} \frac{\mathrm{d} I_{2}}{\mathrm{~d} t}$

$e_{2}=\frac{\mathrm{d} \Phi_{2}}{\mathrm{~d} t}=\frac{\partial \Phi_{2}}{\partial I_{1}} \frac{\mathrm{d} I_{1}}{\mathrm{~d} t}+\frac{\partial \Phi_{2}}{\partial I_{2}} \frac{\mathrm{d} I_{2}}{\mathrm{~d} t}=M^{\prime} \frac{\mathrm{d} I_{1}}{\mathrm{~d} t}+L_{2}^{\prime} \frac{\mathrm{d} I_{2}}{\mathrm{~d} t}$

La méthode consiste à alimenter les circuits avec un générateur continu stabilisé en courant $(\mathrm{d} I / \mathrm{d} t=0)$ et un générateur de courant en forme de rampe $(\mathrm{d} I / \mathrm{d} t=a$ constant $)$ et à mesurer les tensions aux bornes de ces circuits. Si par exemple on fait $\mathrm{d} I_{1} / \mathrm{d} t=a$ et $\mathrm{d} I_{2} / \mathrm{d} t=0$ les équations ci-dessus donnent $L^{\prime}=e_{1} / a$ et $M^{\prime}=e_{2} / a$. Il suffit donc de déterminer comme précédemment $e_{1}(t)$ et $e_{2}(t)$ à partir des tensions $V_{1}(t)$ et $V_{2}(t)$ aux bornes des circuits.

6. Mesures expérimentales sur nos lentilles. - Nous avons appliqué la méthode de mesure décrite ci-dessus à l'étude des coefficients d'autoinduction de lentilles magnétiques à enroulements excitateurs supraconducteurs, les mesures étant effectuées sur ces lentilles en boucle ouverte.

6.1 Alimentation en COURANT. - Nous présentons sur la figure 11 un schéma de principe à commande manuelle du montage qui permet d'obtenir la rampe de courant. Les amplificateurs $A_{1}$ et $A_{2}$ de gains respectifs $(-1)$ et $(+1)$ permettent d'obtenir des signaux carrés commandés par le commutateur $\mathrm{K}$. Ces signaux sont appliqués à l'entrée de l'intégrateur $\mathrm{A}_{3}$ qui délivre une tension triangulaire qu'on applique à l'entrée de $\mathrm{A}_{4}$, amplificateur en tension de l'alimentation en courant. Nous avons réalisé sur ce principe un générateur particulièrement adapté à l'alimentation à forte intensité des enroulements supraconducteurs de nos lentilles, qui permet d'obtenir des rampes de courant dont le maximum peut aller jusqu'à 60 ampères.

6.2 Mesures EFFECTUÉEs. - La géométrie des lentilles que nous avons étudiées est présentée sur la figure 12. Ce sont des lentilles magnétiques dont les carcasses sont en fer doux et dont les enroulements excitateurs sont réalisés en fil supraconducteur $\mathrm{Nb}-\mathrm{Ti}$ de diamètre $33 / 100 \mathrm{~mm}$. Ces lentilles sont disposées dans un cryostat à hélium liquide. Les mesures s'effectuent dans les conditions d'un circuit

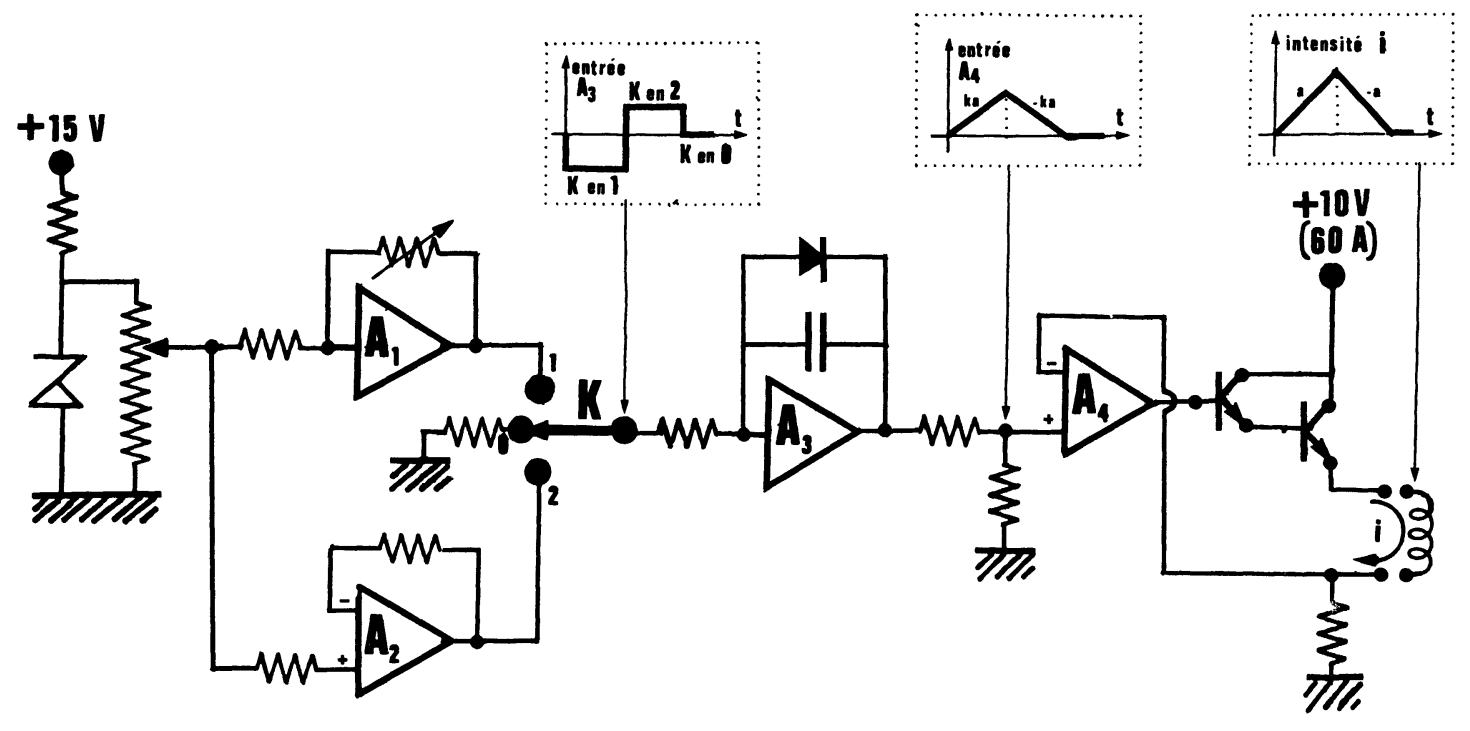

Fig. 11. - Schéma de principe du générateur fournissant la rampe de courant à forte intensité.

[Schematic diagram of the high current supply.] 


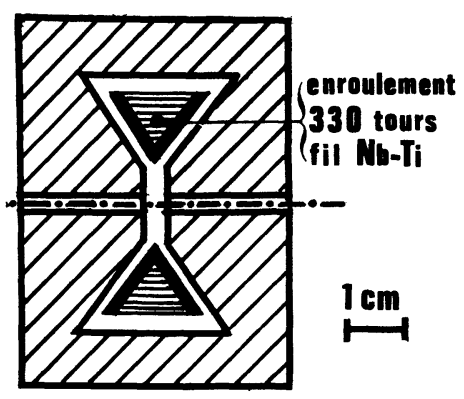

Fig. 12. - Lentilles magnétiques supraconductrices étudiées : coupe méridienne de la lentille (I).

[Studied superconducting lenses : cross-sectionnal diagram of the lens (I).]

sans résistance : il faut donc faire attention aux résistances de contact et pour ne pas faire intervenir la résistance des amenées de courant la tension de l'enroulement doit être prise dans le cryostat aux bornes mêmes du supraconducteur.

Pour chaque lentille nous avons mesuré l'autoinductance propre $L_{\mathbf{p}}$ de l'enroulement seul et l'autoinductance dynamique $L^{\prime}$ de la lentille complète. Nous avons étudié la variation du coefficient dynamique $L^{\prime}$ en fonction de l'excitation de la lentille et nous avons également étudié l'influence de la réluctance de son entrefer que nous avons fait varier. Nous avons enfin étudié expérimentalement la règle de variation de $L^{\prime}$ quand on passe d'une lentille donnée à une lentille semblable dans un rapport $k$. En optique électronique en effet pour étudier les caractéristiques d'une lentille on travaille souvent sur un modèle semblable de dimensions plus petites.

Nous présentons tout d'abord les résultats obtenus pour une lentille que nous appelerons lentille (I) dont le diamètre extérieur était de $60 \mathrm{~mm}$ et dont l'enroulement comportait 330 tours. Sur la figure 13 nous donnons la rampe de courant et la courbe

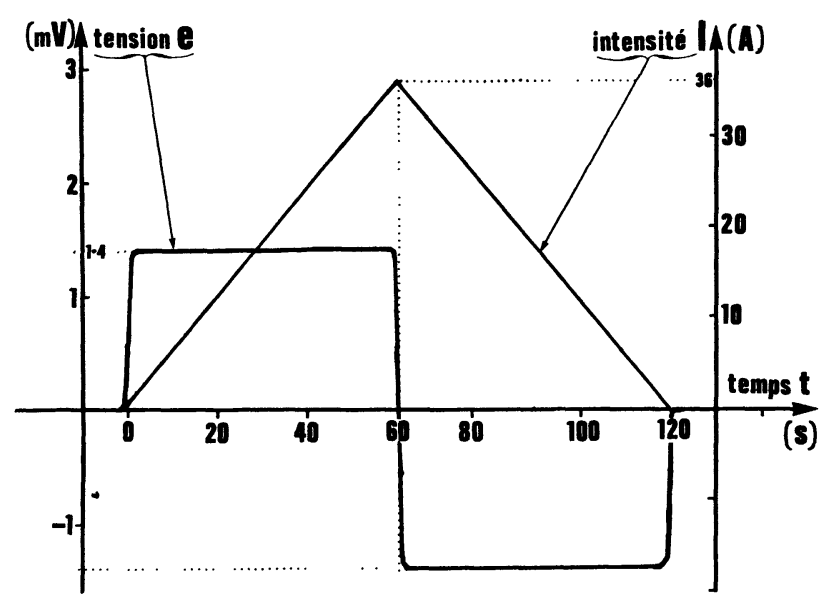

FIG. 13. - Rampe de courant $I(t)$ et tension $e(t)$ enregistrée avec l'enroulement de la lentille (I) seul.

[Current $I(t)$ and induced voltage $e(t)$ in the lens (I) winding without its iron core.] $e(t)$ enregistrée pour l'enroulement seul. Sur la figure 14 nous donnons les mêmes courbes pour la lentille complète avec un entrefer de $5 \mathrm{~mm}$. Sur la figure 15 nous présentons les courbes qu'on en déduit et qui

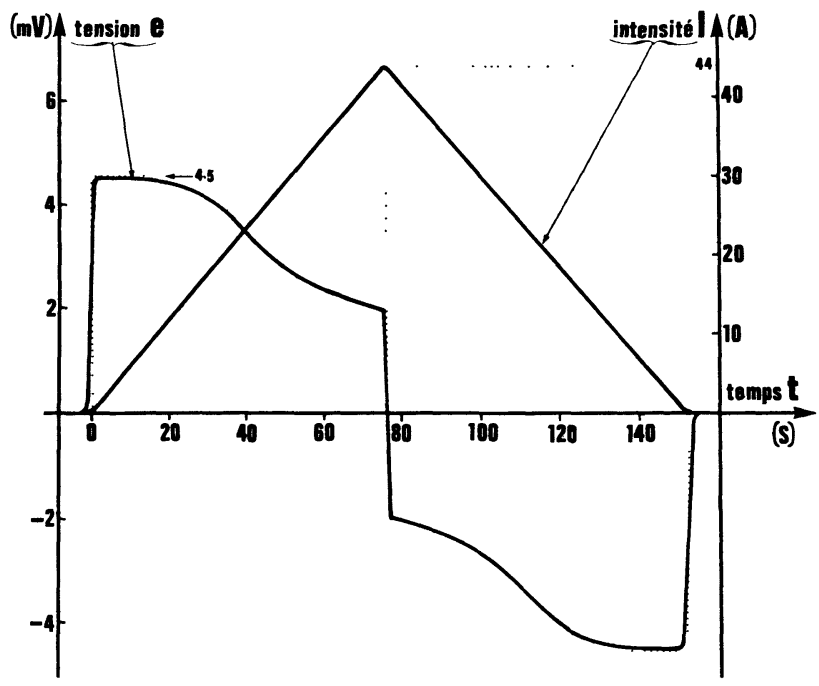

FIG. 14. - Rampe de courant $I(t)$ et tension $e(t)$ enregistrée avec la lentille (I) complète.

[Current $I(t)$ and induced voltage $e(t)$ in the whole lens (I).]

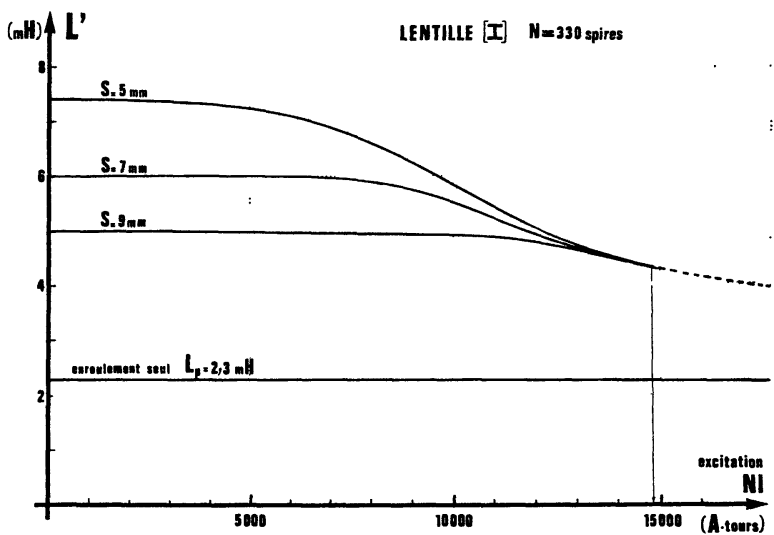

Fig. 15. - Variation du coefficient dynamique d'autoinduction $L^{\prime}$ de la lentille (I) en fonction de son excitation $N I$ et pour diverses valeurs de son entrefer $S$.

[Incremental self-induction coefficient $L^{\prime}$ of the lens (I) versus its excitation $N . I$ for some values of its air-gap $S$.]

donnent la variation de $L^{\prime}$ avec l'excitation $N I$ de la lentille : nous avons regroupé les courbes pour diverses valeurs de l'entrefer et la droite-asymptote qui correspond à l'autoinductance propre $L_{\mathrm{p}} \simeq 2,3 \mathrm{mH}$ de l'enroulement seul. On peut voir qu'avec un entrefer normal de $5 \mathrm{~mm}$, quand on excite la lentille au maximum aux environs de $15000 \mathrm{~A}$. t, l'autoinductance $L^{\prime}$ passe de 7,5 à 4,5 $\mathrm{mH}$. On peut voir également qu'un doublement de l'entrefer de 5 à $10 \mathrm{~mm}$ détermine une variation de $L^{\prime}$ du même ordre de grandeur.

Nous avons ensuite étudié quelques lentilles sem- 
blables à la lentille (I) dans des rapports $k$ supérieurs à 1 : ces lentilles avaient des dimensions géométriques linéaires $k$ fois plus grandes et étaient soumises à des excitations semblables $k . N . I$. La section de leurs enroulements étaient $k^{2}$ fois plus grande et ces enroulements réalisés avec le même fil supraconducteur comportaient donc $k^{2}$ fois plus de tours.

Pour ces lentilles semblables l'excitation maximum possible correspondant au courant maximum était $\left(k^{2} \cdot N\right) \cdot I_{\max }$ soit $k$ fois plus que l'excitation maximum semblable $k \cdot\left(N I_{\max }\right)$.

Par ailleurs, les coefficients d'induction variant comme le carré du nombre de tours des enroulements, on a déjà un coefficient $k^{4}$ qui intervient entre la lentille (I) et les lentilles semblables. Indépendamment de ce premier effet nous souhaitions vérifier expérimentalement que dans une lentille semblable avec un même nombre de tours, la similitude intervenait par un facteur multiplicatif $k$. Dans notre cas nous escomptions donc au total un facteur $k^{5}$ entre le coefficient d'autoinduction $L^{\prime}$ de la lentille (I) et celui des lentilles semblables.

Nous avons en particulier étudié la lentille (II) pour laquelle le rapport de similitude $k$ était égal à 2 . Sur la figure 16 nous présentons la variation de son coefficient $L^{\prime}$ en fonction de son excitation. $\mathrm{Si}$ on compare ces courbes à celles de la figure 15 on peut voir que pour des excitations semblables, soit ici pour des excitations doubles de celles de la lentille (I), les coefficients d'autoinductance sont à pau près 32 fois plus grands que ceux de la lentille (I) : le nombre de tours 4 fois plus grand intervient par un coefficient 16 et la similitude intervient par le coefficient de similitude $k=2$. Sur la figure 16 nous avons tracé en trait pointillé la courbe $S=5 \mathrm{~mm}$ de la lentille (I) (Fig. 15) et qui correspond à la courbe $S=10 \mathrm{~mm}$ de la lentille (II) (Fig. 16). L'écart relatif entre les deux courbes est de l'ordre de $5 \%$ à faible excitation et se réduit à forte excitation. Avec la lentille (I) l'excitation maximum possible était de l'ordre de 15000 A. $\mathrm{t}$ : avec la lentille (II) l'excitation maximum semblable était donc $30000 \mathrm{~A}$. $\mathrm{t}$, cependant que l'excitation maximum possible était $60000 \mathrm{~A}$. $\mathrm{t}$.

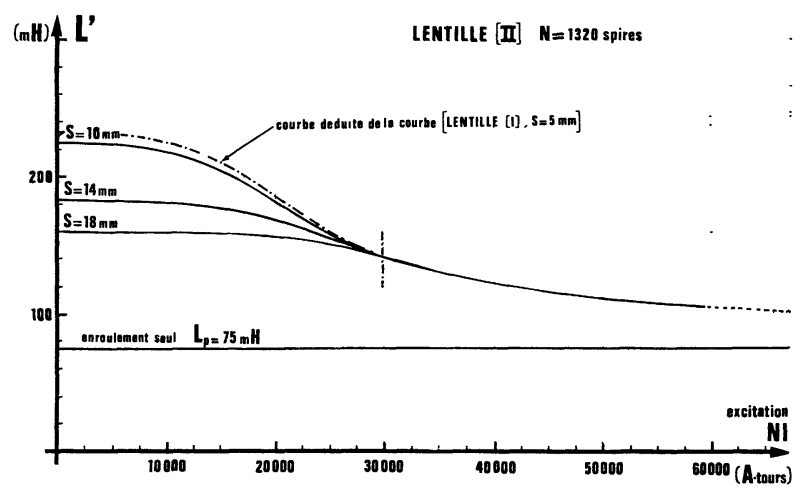

FIG. 16. - Courbes $L^{\prime}(N I)$ pour diverses valeurs de $S$ dans le cas de la lentille (II) semblable à la lentille (I) dans un rapport $k=2$.

[Same curves as in the figure 15 in the case of the lens (II) geometrically similar to the lens (I) (coefficient of similarity $k=2$ ).]

Avec cette lentille (II) nous avons donc pour la courbe $L^{\prime}(N I)$ une portion asymptotique plus importante qui fait mieux apparaître comment la valeur de $L^{\prime}$ s'approche de $L_{\mathrm{p}}$ à forte excitation.

On retiendra également du point de vue des ordres de grandeur que sur toute la gamme d'utilisation d'une lentille, la valeur de son coefficient dynamique d'autoinduction $L^{\prime}$ est comprise approximativement entre 3. $L_{\mathrm{p}}$ et $L_{\mathrm{p}}, L_{\mathrm{p}}$ étant le coefficient d'autoinduction de l'enroulement excitateur de la lentille.

7. Conclusion. - Nous avons présenté une méthode de mesure qu'on peut appliquer à des circuits supraconducteurs ou normaux et qui permet la détermination directe de coefficients dynamiques d'induction. Nous l'avons appliquée dans de bonnes conditions au cas de nos lentilles supraconductrices pour déterminer la valeur de leurs coefficients dynamiques d'autoinduction nécessaire pour prévoir leur fonctionnement en boucle fermée. La méthode est cependant plus générale et pourra être utilisée dans bien d'autres cas en particulier dans le domaine industriel pour des mesures sur des machines électriques ou des transformateurs.

\section{Bibliographie}

[1] Severin, C., Genotel, D., Girard, M., Laberrigue, A., Revue Phys. Appl. 6 (1971) 459.

[2] Genotel, D., Balossier, G., Revue Phys. Appl. 10 (1975) 443.

[3] Prescott, J. C., El-Karashi, A. K., Proc. Inst. Elect. Engrs, 106 pt A (1959) 169.
[4] Barton, T. H., Dunfield, J. C., I. E. E. E. Trans. Power Apparatus and Systems 85 (1965) 145.

[5] SNIDER, L. A., SMIth, I. R., J. Phys. E : Scient. Instr. 4 (1971) 733.

[6] Golding, E. W., Electrical Measurements and Measuring Instruments (3rd ed., Pitman, London). 